
Transplant Surgery

For the purpose of this article, the words "cosmetic" and "aesthetic" will be used interchangeably.

At the 26th World Congress in Hollywood, the results were presented of a poll that was sent to all European ISHRS members regarding whether there were laws or medical guidance in their country clarifying whether Strip Follicular Unit Transplantation (Strip FUT) and Follicular Unit Excision (FUE) are considered to be surgical procedures and who is allowed to perform them-only doctors, only doctors from certain training backgrounds, or other professionals. If it was thought that neither laws nor medical guidance existed, then opinion was sought. Forty-three responses were received from 16 of the 34 European CEN member countries. Since not only was there a great deal of variation in the responses between countries but also opposing answers from respondents within individual countries, it is clear that there is widespread lack of clarity by ISHRS members regarding current European regulation. This poll was devised and distributed to help inform a debate on whether hair transplantation should be included in a European standard for surgical or non-surgical aesthetic procedures.

However, in last month's editor's note to this column (Vol. 28, No. 5; p. 193), Dr. Andreas Finner raised the valid point about whether hair transplant surgery should be considered a cosmetic procedure at all.

For example, Medscape defines androgenetic (or pattern) alopecia as "a genetically determined disorder characterized by the gradual conversion of terminal hairs into indeterminate, and finally into vellus, hairs. It is an extremely common disease that affects men and women." ${ }^{1}$ The International Classification of Diseases (ICD) 2018-2019 American version ICD-10-CM has a diagnosis code for androgenetic alopecia that is L64.9, and a back reference to L00-L99 that is disease of the skin and appendages. ${ }^{2}$

So is hair transplant surgery really a cosmetic surgical procedure if it is used to treat a disease? Cosmetic surgery is defined in England by the Royal College of Surgeons as "an operation, or invasive medical procedure, to alter one's physical appearance for aesthetic rather than medical reasons." ${ }^{\prime \prime}$ Shouldn't treating a disease be considered a medical reason for doing surgery?

The American Society of Plastic Surgeons lists hair transplant surgery as a cosmetic procedure, ${ }^{4}$ however, the American College of Surgery states that "the goal of the cosmetic surgeon is to make changes in body shape or rejuvenate areas that have been affected by aging." ${ }^{\prime 5}$ Androgentic alopecia is not an intrinsic part of aging. Yes, it becomes more common with age, but it is not an inevitable part of

\section{Reflective Questions:}

Do I consider androgenetic alopecia to be a medical condition rather than an aesthetic one, and if so, do I therefore consider hair transplant surgery for male pattern hair loss to be a noncosmetic surgical procedure?

Are there hair loss conditions where I am uncertain whether surgical hair restoration is cosmetic or non-cosmetic?

aging like wrinkles and sagging skin. Cancers become more common with increased age, but one would not say they are part of aging, and so in the same way, losing hair from androgenetic alopecia is not part of aging. By the definition above, if hair transplant surgery is not used to rejuvenate areas that have been affected by aging, is it really the remit of a cosmetic surgeon?

It is accepted that androgenetic alopecia is a progressive condition that is genetically determined and is mediated by male hormones, hence the name. This is different from male beard hypotrichosis that, although it is genetically determined and hormonally mediated, is not progressive and could hardly be considered a disease. A congenital high hairline is also genetically determined but not progressive and is not a medical condition. It is hard to justify hair transplant surgery to thicken low density, patchy beards or to lower hairlines as anything other than a truly cosmetic procedure.

What then about traction alopecia from weaves, braids, tight hairstyles, or turban wearing in Sikh men? Should treating these with hair transplant surgery be considered cosmetic or non-cosmetic?

What about treating hair loss from trichotillomania, which is clearly a psychological condition?

If you agree that hair transplant surgery for compulsive hair pulling is not a cosmetic procedure, then what about overplucked eyebrows, which is a form of hair pulling but not usually from an obsessive-compulsive disorder?

There is likely to be little argument from those within the field that hair restoration surgery to areas of secondary scarring alopecias from burns, trauma, and surgery is a reconstructive and not a cosmetic procedure.

\section{So why is any of this relevant?}

In some countries where there are publicly funded health services, some treatments are not covered. In the UK, for example, cosmetic surgery is not publicly funded and hair transplantation for all indications, including burn scar alopecia, is generally considered to be a cosmetic procedure so is not funded.

Also, in some countries, a tax is added to surgical procedures that are considered cosmetic or aesthetic in nature. In the current climate of health tourism with patients traveling abroad to seek cheap hair transplants where standards and 
safety may be suboptimal, if additional costs are applied to reputable hair transplant clinic procedures through taxation, then this trend is likely to increase.

The World Health Organization defines health as a "state of complete physical, mental, and social well-being and not merely the absence of disease or infirmity." ${ }^{\prime \prime}$

If someone is distressed by their hair loss, then this can cause them significant psychological morbidity that is treatable. In restoring hair loss, a doctor is treating them to restore their mental health regardless of whether the procedure is regarded as cosmetic or non-cosmetic.

In those countries where, for certain conditions, hair restoration could be provided by public funding, this should not be prevented by a misconception that it is a cosmetic procedure.

In regions where patients have to pay for hair restoration, it should not cost them more because there is a misconception that it is a cosmetic procedure and therefore attracts taxation.

In several areas where there is controversy, the ISHRS has issued position statements that can aid its international members with local regulation and legislation and also help to guide public awareness. Perhaps declaring in which circumstances hair transplant surgery should be considered a cosmetic procedure is also an area that merits debate in order to gain consensus.

\section{References}

1. https://emedicine.medscape.com/article/1070167-overview

2. https://www.icd10data.com/ICD10CM/Codes/L00-L99/L60-L75/ L64-/L64.9

3. file:///C:/Users/greg/Downloads/Professional\%20Standards $\% 20$ for $\% 20$ Cosmetic\%20Surgery\%20web\%20(17).pdf file:///C:/1\%20 Downloads/Professional\%20Standards \%20for\%20Cosmetic $\% 20$ Surgery\%20web.pdf, p. 2.

4. https://www.plasticsurgery.org/

5. https://www.facs.org/education/resources/residency-search/ specialties/plastic

6. https://www.who.int/about/mission/en/

\section{ERTIP} Hair Transplant Instruments

For the past 12 years, Ertip Medical has been a leading company for hair transplant and surgical instruments. Our FUE Motorised System helps improve FUE technique. We are CE certified and produce Sharp/Dull FUE motorised or manual punches. We aim to provide our customers with excellent service.

Ertip offers a basic Must Need Set or a Full Set for hair transplantation and specials for wholesalers. We offer fast, worldwide delivery service.

For more details about our instruments, Halide Edip Adıvar Mah. Akar Cad. No:38 Sisli 34382 ISTANBUL TURKEY

call us at +90 2122103220 \& GSM +90 5077656095

Ertip Medical instruments are

Made using the latest technology

* Of the highest quality, and

$$
\text { our full product Budget-friendly. }
$$

line online!
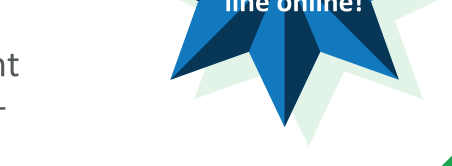